\title{
GMR
}

\section{Aldehyde dehydrogenase 2 protects human umbilical vein endothelial cells against oxidative damage and increases endothelial nitric oxide production to reverse nitroglycerin tolerance}

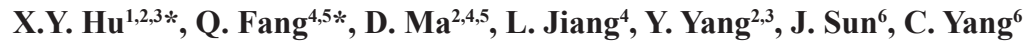 \\ and J.S. Wang ${ }^{1,2,3}$ \\ ${ }^{1}$ Department of Hematology, Affiliated Hospital of GuiZhou Medical University, \\ Guiyang, China \\ ${ }^{2}$ Key Laboratory of Hematological Disease Diagnostic \& Treat Centre of \\ GuiZhou Province, Guiyang, China \\ ${ }^{3}$ GuiZhou Province Hematopoietic Stem Cell Transplantation Center, \\ Affiliated Hospital of GuiZhou Medical University, Guiyang, China \\ ${ }^{4}$ Department of Pharmacy, \\ Affiliated BaiYun Hospital of GuiZhou Medical University, China \\ ${ }^{5}$ Department of Pharmacy, Affiliated Hospital of GuiZhou Medical University, \\ Guiyang, China \\ ${ }^{6}$ School of Pharmacy, GuiZhou Medical University, Guiyang, China \\ *These authors contributed equally to this study. \\ Corresponding authors: Q. Fang / J.S. Wang \\ E-mail: fq_fangqin@sina.com / wangjishi9646@163.com \\ Genet. Mol. Res. 15 (2): gmr. 15027822 \\ Received October 14, 2015 \\ Accepted February 26, 2016 \\ Published June 10, 2016 \\ DOI http://dx.doi.org/10.4238/gmr.15027822
}

ABSTRACT. Medical nitroglycerin (glyceryl trinitrate, GTN) use is limited principally by tolerance typified by a decrease in nitric oxide (NO) produced by biotransformation. Such tolerance may lead to 
endothelial dysfunction by inducing oxidative stress. In vivo studies have demonstrated that aldehyde dehydrogenase 2 (ALDH2) plays important roles in GTN biotransformation and tolerance. Thus, modification of ALDH2 expression represents a potentially effective strategy to prevent and reverse GTN tolerance and endothelial dysfunction. In this study, a eukaryotic expression vector containing the $A L D H 2$ gene was introduced into human umbilical vein endothelial cells (HUVECs) by liposome-mediated transfection. An indirect immunofluorescence assay showed that ALDH2 expression increased $24 \mathrm{~h}$ after transfection. Moreover, real-time polymerase chain reaction and western blotting revealed significantly higher ALDH2 mRNA and protein expression in the gene-transfected group than in the two control groups. GTN tolerance was induced by treating HUVECs with $10 \mu \mathrm{M}$ GTN for $16 \mathrm{~h}+$ 10 min, which significantly decreased NO levels in control cells, but not in those transfected with $A L D H 2$. Overexpression of ALDH2 increased cell survival against GTN-induced cytotoxicity and conferred protection from oxidative damage resulting from nitrate tolerance, accompanied by decreased production of intracellular reactive oxygen species and reduced expression of heme oxygenase 1. Furthermore, ALDH2 overexpression promoted Akt phosphorylation under GTN tolerance conditions. ALDH2 gene transfection can reverse and prevent tolerance to GTN through its bioactivation and protect against oxidative damage, preventing the development of endothelial dysfunction.

Key words: Aldehyde dehydrogenase 2; Oxidative damage; Transfection; Nitric oxide; Nitroglycerin tolerance; Bioconversion

\section{INTRODUCTION}

For more than a century, nitrates have been used as exogenous nitric oxide (NO) donors in cardiovascular disease treatment. As a nitrate drug, nitroglycerin (glyceryl trinitrate, GTN) causes vasodilation through NO-mediated activation of soluble guanylate cyclase and cyclic guanosine monophosphate (cGMP; Madhusoodanan and Murad, 2007; Li et al., 2009). Nevertheless, continuous use of GTN results in rapid development of tolerance, partly due to decreased NO production, and causes serious clinical issues including reduced effectiveness and endothelial dysfunction (Munzel, 2008). Chronic GTN treatment leading to tolerance reduces basal and agonist-stimulated vascular NO bioavailability, which may partly be attributed to abnormal NO generation and NO synthase (NOS) function (Munzel et al., 2005). The resulting endothelial dysfunction predicts adverse long-term outcomes in patients with coronary artery disease. It is now widely believed that bioactivation of GTN is essential for tolerance. A number of enzymatic pathways catalyzing GTN bioactivation have been implicated in nitrate tolerance, such as those involving aldehyde dehydrogenase 2 (ALDH2; McGuire et al., 1998; Doel et al., 2001; Meah et al., 2001; Tsou et al., 2009; Ferreira and Mochly-Rosen, 2012).

As an enzyme responsible for biotransformation of therapeutic doses of GTN, ALDH2 catalyzes GTN into 1,2-glycerol dinitrate and nitrite. Griesberger et al. (2011) reported that 
pharmacological inhibition of this enzyme or deletion of the $A L D H 2$ gene leads to significant tolerance and cross-tolerance, impairing NO-induced vasorelaxation. In addition, treatment with an ALDH inhibitor attenuates the increase in forearm blood flow caused by GTN infusion, indicating that the ALDH pathway contributes to the hemodynamic effects of GTN in humans. ALDH2 seems to play a major role in the development of tolerance and cross-tolerance in vivo. However, it remains unclear whether ALDH2 overexpression can participate in the prevention and reversal of GTN resistance in endothelial cells to relieve endothelial dysfunction.

Reactive oxygen species (ROS) are generated upon development of GTN tolerance. ROS likely inhibit nitrate biotransformation by oxidizing the thiol group of ALDH2 or key coenzymes involved in ALDH activity (Daiber et al., 2009). As a result, GTN biotransformation is compromised, thus inducing nitrate accumulation in mitochondria and aggravating oxidative stress, thereby forming a vicious cycle eventually resulting in endothelial dysfunction and GTN ineffectiveness (Daiber et al., 2009). ALDH2, the main mitochondrial isoform of the ALDH superfamily, is also a key isozyme preventing cellular injury, partly through detoxification of toxic substances such as the reactive aldehyde 4-hydroxynonenal (4-HNE). By metabolizing and scavenging 4-HNE in the inner mitochondrial membrane, ALDH2 prevents oxidative stress-induced cellular damage and maintains the metabolic equilibrium of ROS in vivo, thus protecting cells from injury by oxidation products (Ohsawa et al., 2003). Our group has also verified that high ALDH2 expression confers resistance to oxidative damage (Hu et al., 2011). Moreover, overexpression of this enzyme decreases ROS and activates the ERK/MAPK and PI3K-Akt pathways to limit tissue hyperoxia-induced cell death, accompanied by changes in heme oxygenase 1 (HO-1) expression in the anti-oxidative stress system in vivo (Ohta and Ohsawa, 2006). Nevertheless, the ability of ALDH2 to reverse GTN tolerance and protect endothelial cells via the abovementioned pathways remains to be examined in detail. The aim of this study was to determine whether overexpression of ALDH2 can protect human umbilical vein endothelial cells (HUVECs) against GTN-induced cytotoxicity, accelerate GTN bioactivation, and prevent GTN tolerance. The role of oxidative stress in these processes and the prevention of endothelial dysfunction were also studied.

\section{MATERIAL AND METHODS}

\section{Drugs and reagents}

3-(4,5-Dimethylthiazol-2-yl)-2,5-diphenyltetrazolium bromide (MTT) was purchased from Guizhou ScienceLab Instrument Inc. (Guiyang, China). Dulbecco's modified Eagle's medium (DMEM) and fetal bovine serum (FBS) were supplied by Gibco (Waltham, MA, USA). TRIzol reagent, Lipofectamine 2000, and pcDNA3.1(+) were manufactured by Invitrogen Life Technologies (Carlsbad, CA, USA). Unless otherwise stated, all antibodies were purchased from Abnova (Taiwan, China), while the pBS-T vector was supplied by TIANGEN (Beijing, China).

\section{Cell culture}

HUVECs were a generous gift from the Laboratory of Immunology, Guiyang Medical College, China. This study was approved by the ethics committee of Guiyang Medical College. $A L D H 2, H O-1$, and $\beta$-actin primers were synthesized by Nanjing GenScript Biotech Co., 
Ltd. (Nanjing, China). HUVECs were cultured in DMEM containing 10\% FBS and 0.03\% glutamic acid on six-well plates in a $5 \% \mathrm{CO}_{2}$ atmosphere at $37^{\circ} \mathrm{C}$.

\section{Eukaryotic expression vector construction}

Total RNA was extracted from normal human hepatocytes and used to synthesize ALDH2 complementary DNA (cDNA) by reverse transcription polymerase chain reaction (PCR). cDNA was then cloned into the pBS-T vector with PrimeSTAR HS DNA polymerase (TaKaRa, Dalian, China). The following PCR primers, each containing a restriction enzyme site, were used: sense, 5'-GAC ACG AAT TCA TGT TGC GCG CTG CCG CCC GCT TCG G-3'; and antisense, 5'-GAC ACG GAT CCT TAT GAG TTC TTC TGA GGC ACT TTG AC-3'. pBST-ALDH2 and pcDNA3.1(+) were simultaneously digested with the restriction endonucleases EcoRI and BamHI. To construct the eukaryotic expression plasmid pcDNA3.1(+)-ALDH2, the linearized pcDNA3.1(+) plasmid and purified $A L D H 2$ fragments were ligated by T4 DNA ligase at a ratio of 2:1. pcDNA3.1(+)-ALDH2 was subsequently identified by endonuclease digestion and PCR, and sent to Jinsite Bio Inc. (Nanjing, China) for sequencing.

\section{$A L D H 2$ transfection and analysis of ALDH2 expression}

HUVECs were transfected with pcDNA3.1(+)-ALDH2 using the Lipofectamine 2000 transfection reagent. The pcDNA-CON group was transfected with empty pcDNA3.1(+) plasmids, while the non-transfected blank group was used as a negative control. ALDH2 protein expression was measured by indirect immunofluorescence at 12, 24, 48, and $72 \mathrm{~h}$ after transfection. Relative transfection efficiency was calculated by observing fluorescence intensity.

TRIzol reagent was used to extract total RNA, and $A L D H 2$ mRNA expression was detected by real-time PCR with the following primers: GAPDH upstream, 5'-GAA GGT GAA GGT CGG ATG C-3' and downstream, 5'-GAA GAT GGT GAT GGG ATT TC-3' (262-bp product); ALDH2 upstream, 5'-GAT TTG GAC AAG GCC AAT TAC CTG-3' and downstream, 5'-AAG GGT GAC TGG GCT CCA AAC-3' (217-bp product). Real-time PCR conditions were as follows: pre-denaturation at $94^{\circ} \mathrm{C}$ for $2 \mathrm{~min} ; 35$ cycles of denaturation at $94^{\circ} \mathrm{C}$ for $10 \mathrm{~s}$, annealing at $58^{\circ} \mathrm{C}$ for $20 \mathrm{~s}$, and extension at $68^{\circ} \mathrm{C}$ for $60 \mathrm{~s}$. Relative expression of the target gene was calculated by the $2^{-\Delta \Delta C t}$ method with $G A P D H$ as the internal reference.

Using $10 \%$ sodium dodecyl sulfate-polyacrylamide gel electrophoresis, equal amounts $(20 \mu \mathrm{g})$ of protein extract were separated and transferred to polyvinylidene fluoride membranes. The blotted membranes were blocked with 5\% skim milk in Tris-buffered saline (TBS), and probed with ALDH2 monoclonal antibody (1:250; H00000217-M01) for $1.5 \mathrm{~h}$. After three 10 -min washes with TBS containing $0.05 \%$ Tween 20 , membranes were incubated at room temperature for $1.5 \mathrm{~h}$ with horseradish peroxidase-conjugated goat anti-mouse IgG secondary antibody. Antibody binding was visualized using an enhanced chemiluminescence (ECL) detection kit (Santa Cruz Biotechnology, Dallas, TX, USA) following the manufacturer protocol. Relative ALDH2 protein expression was calculated with ImageJ software v4.0.

\section{Cytotoxicity assay}

GTN-induced cytotoxicity in cells of the three groups was examined using the MTT test. Briefly, cells were seeded at a density of $5 \times 10^{3}$ cells/well on 96-well plates and incubated 
overnight. The culture medium was then discarded, and media containing $0,0.001,0.01,0.1$, $1,10,100$, and $1000 \mathrm{mM} \mathrm{GTN}$ were added to the cells. Tests were performed at least four times for each concentration. Seventy-two hours later, up to $20 \mu \mathrm{L}$ MTT $(5 \mathrm{mg} / \mathrm{mL})$ was added and incubated with cells for $4 \mathrm{~h}$. After centrifugation, the MTT-containing medium was discarded before addition of $120 \mu \mathrm{L}$ dimethyl sulfoxide. Optical density at $570 \mathrm{~nm}$ was measured using a plate reader, with a reference wavelength of $485 \mathrm{~nm}$. Analysis of cell viability as a percentage of the untreated controls was performed with SPSS software (SPSS Inc., Chicago, IL, USA) to calculate half maximal inhibitory concentration $\left(\mathrm{IC}_{50}\right)$ values.

\section{NO production}

The rate at which GTN metabolism yielded NO was determined following the protocol of the NO analysis kit manufacturer. Briefly, GTN tolerance was induced in cells of the three groups (pcDNA-ALDH2, pcDNA-CON, and blank) by incubation with $1 \times 10^{-5} \mathrm{M} \mathrm{GTN}$ at $37^{\circ} \mathrm{C}$ for $16 \mathrm{~h}+10 \mathrm{~min}$. The GTN non-tolerance treatment consisted of incubating cells of the same three groups with $1 \times 10^{-5} \mathrm{M}$ GTN at $37^{\circ} \mathrm{C}$ for $10 \mathrm{~min}$. In addition, cells not treated with GTN were used as control groups. NO concentration was quantified by spectrophotometry, and results are expressed as $\mu \mathrm{mol}$ per $1 \times 10^{4}$ cells.

\section{ROS analysis}

Intracellular ROS were measured based on ROS-mediated oxidation of nonfluorescent 2',7'-dichlorofluorescin diacetate (DCFH-DA) into fluorescent DCFH. Cells were seeded $48 \mathrm{~h}$ after transfection. After incubation in the presence of GTN for $16 \mathrm{~h}+10 \mathrm{~min}$ or $10 \mathrm{~min}$, or in its absence, cells were plated at a density of $1 \times 10^{6}$ cells/well on six-well plates and incubated with 10 $\mu \mathrm{M}$ DCFH-DA for $15 \mathrm{~min}$. The conversion of DCFH-DA to DCFH by intracellular ROS was then measured with fluorescence spectrophotometry (Cary Eclipse; Agilent, Santa Clara, CA, USA).

\section{HO-1 mRNA expression}

GTN tolerance and non-tolerance models were established by incubating HUVECs with $1 \times 10^{-5} \mathrm{M}$ GTN for $16 \mathrm{~h}+10 \mathrm{~min}$ or $10 \mathrm{~min}$. Subsequently, cells were collected and $H O-1$ mRNA expression measured by real-time PCR. GAPDH served as an internal reference, and the abovementioned reaction conditions were used with the following primers: $H O-1$ upstream, 5'-CCA GTG CCA CCA AGT TCA AGC-3', and downstream, 5'-AGG CAT GTT GAC TTC ACT T-3' (205-bp product). Relative target gene expression was calculated according to the method above.

\section{Phospho-Akt/Akt protein expression}

To analyze Akt phosphorylation, cells were washed with cold phosphate-buffered saline and treated with GTN for $16 \mathrm{~h}+10 \mathrm{~min}$ or $10 \mathrm{~min}$. Cells were then lysed and the resulting proteins incubated with phospho-Akt/Akt antibody (Cell Signaling Technology, Danvers, MA, USA) overnight. The cells were then washed with TBS, treated with lysis buffer, and scraped from the plate. Most of the steps used to analyze this protein were the same as those given above. 


\section{Data and statistical analysis}

All values are given as means \pm standard deviation. Differences between means were analyzed by the Student's $t$-test. Differences between groups were assessed by analysis of variance.

\section{RESULTS}

\section{ALDH2 protein fluorescence}

Cultured cells on creep plates were incubated with ALDH2 monoclonal antibody and fluorescein isothiocyanate-labeled secondary antibody. Based on fluorescence intensity, pcDNA-ALDH2 group was seen to express ALDH2 protein $24 \mathrm{~h}$ after transfection. This fluorescence became most bright $72 \mathrm{~h}$ after transfection (Figure 1A) and remained higher for at least 1 week. To calculate transfection efficiency, cells with the lowest and highest fluorescence intensities were considered as negative and positive controls, respectively. The transfection efficiency using HUVECs was $11.5 \pm 1.0 \%$ at $24 \mathrm{~h}, 76.5 \pm 3.5 \%$ at $48 \mathrm{~h}$, and $100 \%$ at $72 \mathrm{~h}$ (Figure 1B).
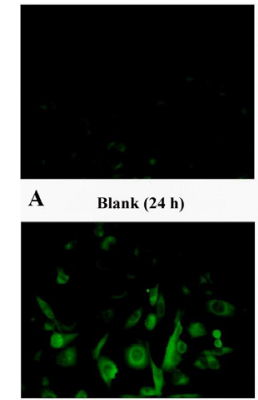

pCDNA-ALDH2 (24 h)

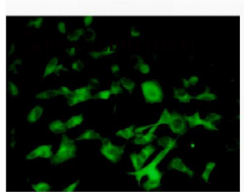

pcDNA-ALDH2 (72 h)

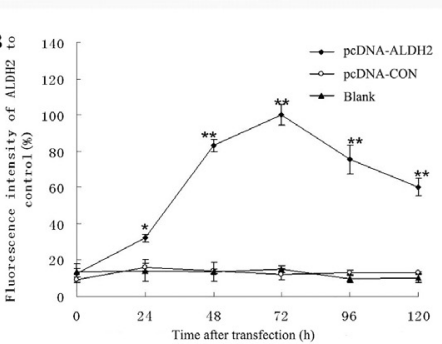

Figure 1. Observation of ALDH2 protein expression in human umbilical vein endothelial cells (HUVECs) by indirect immunofluorescence assay. A. pcDNA-ALDH2 and pcDNA-CON (nonsense) vectors were transfected into HUVECs, and fluorescence was observed after $24 \mathrm{~h}$. B. Fluorescence became most brightat $72 \mathrm{~h} \mathrm{in} \mathrm{pcDNA-}$ ALDH2 cells $(* \mathrm{P}<0.05$ vspcDNA-CON; $* * \mathrm{P}<0.01$ vspcDNA-CON). 


\section{ALDH2 overexpression in HUVECs}

To determine the effect of pcDNA-ALDH2 plasmid transfection on HUVECs, ALDH2 mRNA and protein expression was measured. $A L D H 2$ mRNA levels were significantly higher in the pcDNA-ALDH2 group than the pcDNA-CON and blank control groups $(\mathrm{P}<0.05)$, which did not differ from each other in this regard (Figure 2A). Therefore, ALDH2 mRNA was highly expressed in HUVECs transfected with the $A L D H 2$-carrying plasmid. Western blotting showed that pcDNA-ALDH2 cells expressed significantly higher levels of human ALDH2 protein, seen as a distinct band after ECL visualization, than the two control groups, which produced only faint reactions (Figure $2 \mathrm{~B}$ and $\mathrm{C}$ ). Given this high level of ALDH2 protein expression $72 \mathrm{~h}$ after transfection, it was determined that successful expression of the pcDNA3.1-ALDH2 construct in HUVECs had been achieved.
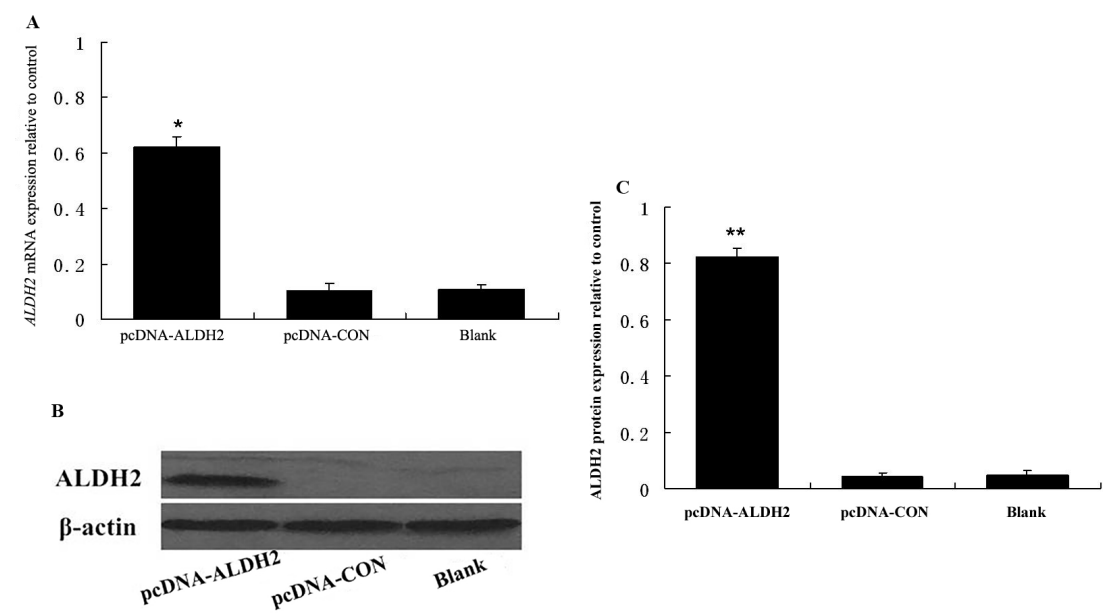

Figure 2. Detection of ALDH2 expression in HUVECs by real-time polymerase chain reaction (PCR) and western blotting. A. Semi-quantitative assay of $A L D H 2$ mRNA expression based on ImageJ software analysis $(* \mathrm{P}<0.05 v s$ pcDNA-CON). B. Western blot of ALDH2 protein levels. C. Semi-quantitative assay of ALDH2 protein expression based on ImageJ software analysis $(* * \mathrm{P}<0.01 v s$ pcDNA-CON).

\section{GTN cytotoxicity and tolerance were inhibited by ALDH2 overexpression}

The relationship between GTN concentration and cell survival was analyzed by MTT assay (Figure 3A). A semi-logarithmic regression equation (calculated with SPSS) revealed GTN IC $_{50}$ values in the pcDNA-ALDH2, pcDNA-CON, and blank groups to be 96.25, 5.31, and $4.16 \mathrm{mM}$, respectively. Thus, the pcDNA-ALDH2 group demonstrated greater tolerance of GTN cytotoxicity than the other groups $(\mathrm{P}<0.01)$, which did not significantly differ from each other $(\mathrm{P}>0.05)$. Compared to a 10-min incubation with GTN, tolerance conditions significantly reduced NO production in the two control groups, but not in pcDNA-ALDH2 cells, which showed comparable levels of NO under both GTN treatments $(\mathrm{P}<0.01$; Figure $3 \mathrm{~B})$. There was no significant difference between the three groups when treated with GTN for 10 min. Therefore, $A L D H 2$ transfection markedly increased NO generation under GTN tolerance conditions. 

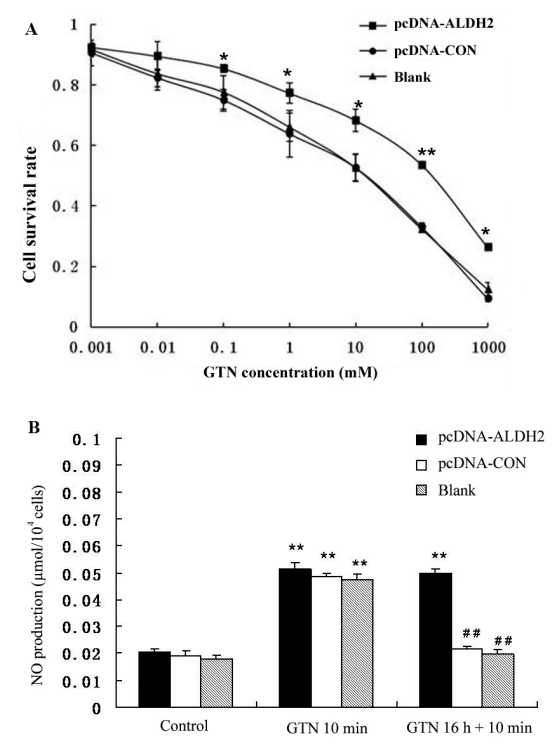

Figure 3. Upregulation of ALDH2 expression inhibits nitroglycerin (glyceryl trinitrate, GTN) cytotoxicity and tolerance. A. Survival of HUVECs transfected with nonsense DNA (pcDNA-CON) or ALDH2 (pcDNA-ALDH2) vectors was measured using a 3-(4,5-dimethylthiazol-2-yl)-2,5-diphenyltetrazolium bromide assay at an optical density of $490 \mathrm{~nm}(* \mathrm{P}<0.05$ vs pcDNA-CON; $* * \mathrm{P}<0.01$ s pcDNA-CON). B. In GTN-resistant cells (GTN 16 $\mathrm{h}+10 \mathrm{~min}$ ), nitric oxide (NO) production was higher in the pcDNA-ALDH2 group, suggesting that ALDH2 may alleviate GTN tolerance at certain concentrations ( ${ }^{\mathrm{P}}<0.01$ vs pcDNA-ALDH2; ** $\mathrm{P}<0.01$ vs Control).

\section{ALDH2 overexpression suppressed oxidative damage in GTN tolerance}

After treatment with GTN for $16 \mathrm{~h}+10 \mathrm{~min}$, ROS levels were significantly lower in pcDNA-ALDH2 cells than the two control groups $(\mathrm{P}<0.01$; Figure $4 \mathrm{~A})$, which did not differ in this respect $(\mathrm{P}>0.05)$. ALDH2 transfection, which substantially decreased intracellular ROS generation, prevented ROS levels from increasing further. Since HO-1 responds to cellular stress and many oxidative stimuli (Daiber et al., 2009), we measured its expression. The HO-1 mRNA was activated and its levels thereafter decreased with increase in ROS following ALDH2 transfection. $H O-1$ mRNA levels in pcDNA-ALDH2 cells under GTN tolerance conditions were significantly lower than those of the control groups, which did not differ from each other with respect to relative $\mathrm{HO}-\mathrm{l}$ expression (Figure 4B).

\section{ALDH2 overexpression activated the Akt signaling pathway in GTN tolerance}

The serine/threonine protein kinase Akt has downstream anti-apoptotic effects when activated by growth factors through PI3K. It has been reported that Akt mediates the activation of endothelial NOS, thus increasing NO production (Cokic et al., 2014). Moreover, ALDH2 stimulates Akt phosphorylation (Ohta and Ohsawa, 2006). The effects of ALDH2 on Akt signaling in GTN tolerance were evaluated. Akt phosphorylation was found to be decreased under GTN tolerance conditions, in agreement with previous reports (Ohta and Ohsawa, 2006), with the exception of ALDH2-transfected cells, in which it was significantly stimulated (Figure 5A and B). 

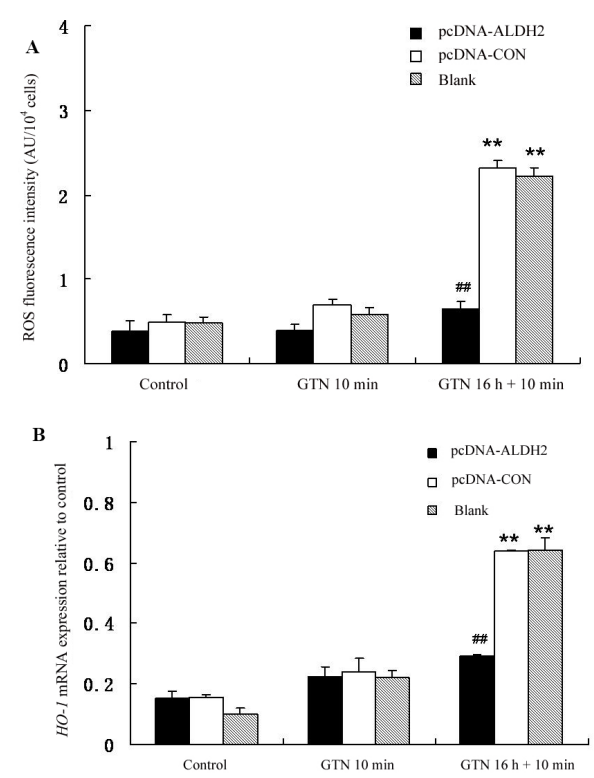

Figure 4. Upregulation of ALDH2 expression suppresses oxidative damage during GTN tolerance. A. Reactive oxygen species (ROS) assay of GTN-tolerant HUVECs transfected with pcDNA-CON or pcDNA-ALDH2 (arbitrary units, $\mathrm{AU})\left({ }^{\#} \mathrm{P}<0.01\right.$ vs pcDNA-CON; $* * \mathrm{P}<0.01$ vs Control). B. Detection of HO-1 expression in HUVECs by real-time PCR. Values shown were calculated from at least three independent experiments ${ }^{\# \#} \mathrm{P}<0.01$ vs pcDNA-CON; ${ }^{* *} \mathrm{P}<0.01$ vs Control).
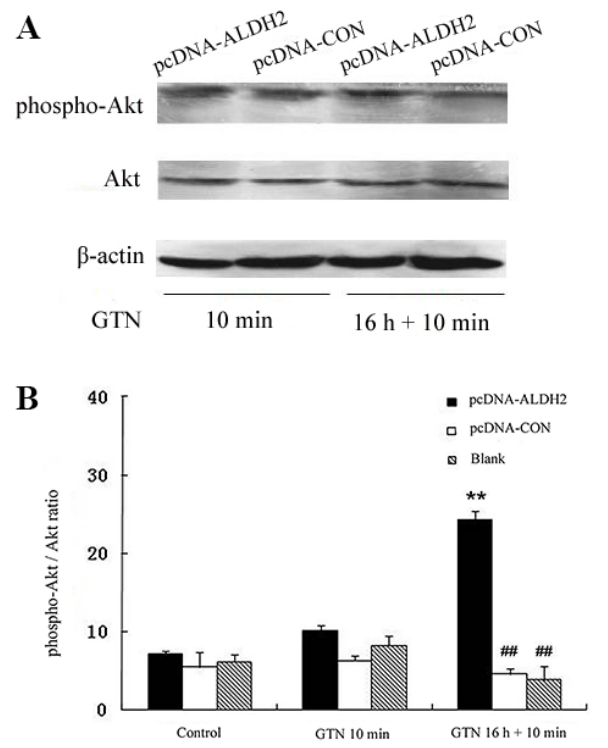

Figure 5. Upregulation of ALDH2 expression stimulated Akt phosphorylation during GTN tolerance. A. and B. Phospho-Akt was normalized to total Akt in HUVECs treated with GTN for $16 \mathrm{~h}+10 \mathrm{~min}$ or $10 \mathrm{~min}\left({ }^{\#} \mathrm{P}<0.01 \mathrm{vs}\right.$ pcDNA-CON; **P $<0.01$ vs Control). 


\section{DISCUSSION}

Organic nitrates, such as GTN, have been widely applied in treating cardiovascular diseases. Although GTN is effective for acute attacks of angina pectoris, continuous or frequent use easily leads to tolerance. Researchers have endeavored to clarify the mechanisms by which GTN functions and induces tolerance. Recently, particular attention has been paid to the decreased ALDH2 activity observed because of GTN tolerance. It is widely recognized that this enzyme strongly affects alcohol metabolism (Huang et al., 2004; Hendershot et al., 2009). Furthermore, ALDH2 plays a significant role in protecting cells from toxic substances (Klyosov et al., 1996; Wang et al., 2009). Feldman and Weiner (1972) reported that ALDH2 demonstrates the activities of both a dehydrogenase and an esterase, which share the same active center. As a matrix enzyme in mitochondria, ALDH2 protects against oxidative damage by attenuating ROS generation and oxidizing exogenous and endogenous aldehydes such as 4-HNE, a product of lipid peroxidation (Luckey and Petersen, 2001). Importantly, ALDH2 has an essential role in GTN bioactivation, which is associated with the formation of NO free radicals, resulting in cGMP-mediated vascular relaxation (Beretta et al., 2008). Earlier reports described the inhibition of ALDH by organic nitrates, but the pharmacological implications were not considered before the discovery of ALDH2-catalyzed GTN bioactivation in 2002 (Chen et al., 2002; Kollau et al., 2005). Zhang et al. (2004) reported that reductants are essential for sustained ALDH2-catalyzed GTN metabolism and bioactivation. Furthermore, Baretta et al. (2008) found that GTN biotransformation was promoted by addition of ALDH2 expressed and purified in vitro. In humans, endothelial cells represent vascular barriers and targets for many drugs and biomolecules. Although the crucial role of ALDH2 in GTN transformation in vivo has been well documented (Mayer and Beretta, 2008; Chang et al., 2012), its underlying mechanism of action and the involvement of other processes, such as oxidative stress, require clarification. In the present study, we established that $A L D H 2$ transfection was sufficient for GTN bioactivation in HUVECs in vitro, and examined the involvement of anti-oxidative stress in prevention of endothelial dysfunction.

Based on its reduced activity and high mutation rate in GTN tolerance, ALDH2 may play a major role in the prevention of tolerance and cross-tolerance. Here, we verified this proposition by showing that $A L D H 2$ overexpression prevents nitrate tolerance in vitro. Although ALDH2 significantly contributes to vascular GTN bioactivation, it is unclear how its activity, yielding inorganic nitrite as the final product, is linked to GTN tolerance in endothelial cells. First, we cloned the $A L D H 2$ gene from normal human hepatocytes and constructed the eukaryotic expression vector pcDNA3.1/myc-His-ALDH2, which was successfully transfected into HUVECs. ALDH2 mRNA and protein were highly expressed in pcDNA-ALDH2 cells. Moreover, compared to the controls, high GTN concentrations induced lower cytotoxicity in the $A L D H 2$-transfected group, most likely due to anti-oxidative stress resulting from high ALDH2 expression. This presumption was confirmed in further experiments.

We then clarified the mechanism underlying the formation of NO free radicals in ALDH-catalyzed GTN metabolism. Since NO generation was significantly enhanced upon high ALDH2 expression, the transfected construct was capable of converting nitrite into NO. Decreased NO in endothelial cells results in endothelial dysfunction, which may accelerate the progression of angiocardiopathy (Kollau et al., 2009). In the current work, a GTN tolerance model was successfully constructed. Under such conditions, the $A L D H 2$-transfected group showed higher NO production than the control groups, signifying greater ALDH2 activity. 
Moreover, $A L D H 2$ transfection may decrease ROS formation and protect against endothelial dysfunction in GTN tolerance.

In addition, GTN tolerance (Fadel et al., 2012) and corresponding biological and chemical changes (Gori and Parker, 2002; Daiber et al., 2009) have been attributed to ROS-induced oxidative stress. In this study, we observed that more ROS were produced under GTN tolerance conditions. ROS may inhibit nitrate biotransformation by oxidizing the thiol group of ALDH2 or key coenzymes involved in ALDH activity, thus inducing nitrate accumulation in mitochondria, aggravating oxidative stress, and ultimately forming a vicious cycle. ALDH2 overexpression may play a positive role in preventing oxidative stress-induced cell damage. Indeed, ROS levels barely increased in the $A L D H 2$-transfected group after GTN tolerance treatment. From these results, we postulate that chronic GTN application stimulated mitochondrial ROS production, which was suppressed by overexpression of ALDH2. Similar effects may occur during the metabolism of 4-HNE and the accompanying decrease in intracellular ROS (Hu et al., 2011).

During GTN tolerance, oxidative stress stimulates high expression of HO-1 (Daiber and Munzel, 2010). After stimulation of HO-1 with hemin, GTN therapy no longer generates ROS nor induces tolerance. In contrast, treatment with the HO-1 inhibitor apigenin results in GTN tolerance. Similarly, we found that $H O-1$ mRNA levels increased under GTN tolerance conditions. Since $H O-1$ transcription rose only minimally in the $A L D H 2$-transfected group, high ALDH2 levels may confer resistance to oxidative stress by reducing ROS release, leading to negative feedback decreasing $\mathrm{HO}-\mathrm{l}$ expression. Endogenous ALDH2 overexpression promotes activation of the PI3K-Akt pathway. In normal and high oxygen environments, ALDH2 overexpression augments Akt phosphorylation and maintains total Akt levels, thereby facilitating cell growth (Ohta and Ohsawa, 2006). Here, we showed that ALDH2 overexpression was necessary for activation of PI3K-Akt in GTN tolerance, while overexpression of constitutively active Akt was involved in reducing such tolerance. Increased phosphorylation of Akt may be one of the mechanisms by which ALDH2 protects endothelial cells.

In summary, this study provides another novel example of the circumvention of GTN tolerance in HUVECs. Overexpression of ALDH2 in vitro relieved nitrate tolerance and increased NO generation, enabling the biological utilization of GTN. Furthermore, ALDH2 overexpression markedly inhibited tolerance-induced oxidative stress, accompanied by decreased generation of intracellular ROS and reduced expression of $\mathrm{HO}-1$, associated with the Akt signaling pathway. Taken as a whole, ALDH2 contributed to GTN bioactivation and protection of endothelial function against oxidative damage in GTN tolerance. To some degree, ALDH2 played a moderately protective role in endothelial cell function. However, further in vivo studies examining the role of ALDH2 in repairing endothelial dysfunction are required.

\section{Conflicts of interest}

The authors declare no conflict of interest.

\section{ACKNOWLEDGMENTS}

Research supported in part by the National Natural Science Foundation of China (\#81360501, \#81270636, and \#81070444), the Joint Fund of Guizhou Province [\#LH (2014)7130], the Social Development Project [\#SY(2012)3138], and the International Cooperation Project (\#2011-7010) of Guizhou Province. 


\section{REFERENCES}

Beretta M, Gruber K, Kollau A, Russwurm M, et al. (2008). Bioactivation of nitroglycerin by purified mitochondrial and cytosolic aldehyde dehydrogenases. J. Biol. Chem. 283: 17873-17880. http://dx.doi.org/10.1074/jbc.M801182200

Chang YC, Chiu YF, Lee IT, Ho LT, et al. (2012). Common ALDH2 genetic variants predict development of hypertension in the SAPPHIRe prospective cohort: gene-environmental interaction with alcohol consumption. BMC Cardiovasc. Disord. 12: 58. http://dx.doi.org/10.1186/1471-2261-12-58

Chen Z, Zhang J and Stamler JS (2002). Identification of the enzymatic mechanism of nitroglycerin bioactivation. Proc. Natl. Acad. Sci. USA 99: 8306-8311. http://dx.doi.org/10.1073/pnas.122225199

Cokic BB, Cokic VP, Suresh S, Wirt S, et al. (2014). Nitric oxide and hypoxia stimulate erythropoietin receptor via MAPK kinase in endothelial cells. Microvasc. Res. 92: 34-40. http://dx.doi.org/10.1016/j.mvr.2014.01.009

Daiber A and Munzel T (2010). Characterization of the antioxidant properties of pentaerithrityl tetranitrate (PETN)induction of the intrinsic antioxidative system heme oxygenase-1 (HO-1). Methods Mol. Biol. 594: 311-326. http:// dx.doi.org/10.1007/978-1-60761-411-1_22

Daiber A, Oelze M, Coldewey M, Kaiser K, et al. (2005). Hydralazine is a powerful inhibitor of peroxynitrite formation as a possible explanation for its beneficial effects on prognosis in patients with congestive heart failure. Biochem. Biophys. Res. Commun. 338: 1865-1874.http://dx.doi.org/10.1016/j.bbrc.2005.10.106

Daiber A, Oelze M, Wenzel P, Wickramanayake JM, et al. (2009). Nitrate tolerance as a model of vascular dysfunction: roles for mitochondrial aldehyde dehydrogenase and mitochondrial oxidative stress. Pharmacol. Rep. 61: 33-48. http://dx.doi.org/10.1016/S1734-1140(09)70005-2

Doel JJ, Godber BL, Eisenthal R and Harrison R (2001). Reduction of organic nitrates catalysed by xanthine oxidoreductase under anaerobic conditions. Biochim. Biophys. Acta 1527: 81-87. http://dx.doi.org/10.1016/S0304-4165(01)00148-9

Esplugues JV, Rocha M, Nuñez C, Bosca I, et al. (2006). Complex I dysfunction and tolerance to nitroglycerin: an approach based on mitochondrial-targeted antioxidants. Circ. Res. 99: 1067-1075. http://dx.doi.org/10.1161/01. RES.0000250430.62775.99

Fadel PJ, Farias Iii M, Gallagher KM, Wang Z, et al. (2012). Oxidative stress and enhanced sympathetic vasoconstriction in contracting muscles of nitrate-tolerant rats and humans. J. Physiol. 590: 395-407. http://dx.doi.org/10.1113/ jphysiol.2011.218917

Feldman RI and Weiner H (1972). Horse liver aldehyde dehydrogenase. II: Kinetics and mechanistic implications of the dehydrogenase and esterase activity. J. Biol. Chem. 247: 267-272.

Ferreira JC and Mochly-Rosen D (2012). Nitroglycerin use in myocardial infarction patients. Circ. J. 76: 15-21. http:// dx.doi.org/10.1253/circj.CJ-11-1133

Gori T and Parker JD (2002). Nitrate tolerance: a unifying hypothesis. Circulation 106: 2510-2513.

Griesberger M, Kollau A, Wölkart G, Wenzl MV, et al. (2011). Bioactivation of pentaerythrityl tetranitrate by mitochondrial aldehyde dehydrogenase. Mol. Pharmacol. 79: 541-548. http://dx.doi.org/10.1124/mol.110.069138

Hendershot CS, Collins SE, George WH, Wall TL, et al. (2009). Associations of ALDH2 and ADH1B genotypes with alcohol-related phenotypes in Asian young adults. Alcohol. Clin. Exp. Res. 33: 839-847. http://dx.doi.org/10.1111/ j.1530-0277.2009.00903.X

Hu XY, Fang Q, Wang JS, Xie JQ, et al. (2011). Over-expression of aldehyde dehydrogenase-2 protects against $\mathrm{H}_{2} \mathrm{O}_{2^{-}}$ induced oxidative damage and apoptosis in peripheral blood mononuclear cells. Acta Pharmacol. Sin. 32: 245-252. http://dx.doi.org/10.1038/aps.2010.203

Huang SY, Lin WW, Ko HC, Lee JF, et al. (2004). Possible interaction of alcohol dehydrogenase and aldehyde dehydrogenase genes with the dopamine D2 receptor gene in anxiety-depressive alcohol dependence. Alcohol. Clin. Exp. Res. 28: 374-384.http://dx.doi.org/10.1097/01.ALC.0000117832.62901.61

Klyosov AA, Rashkovetsky LG, Tahir MK and Keung WM (1996). Possible role of liver cytosolic and mitochondrial aldehyde dehydrogenases in acetaldehyde metabolism. Biochemistry 35: 4445-4456. http://dx.doi.org/10.1021/bi9521093

Kollau A, Hofer A, Russwurm M, Koesling D, et al. (2005). Contribution of aldehyde dehydrogenase to mitochondrial bioactivation of nitroglycerin: evidence for the activation of purified soluble guanylate cyclase through direct formation of nitric oxide. Biochem. J. 385: 769-777. http://dx.doi.org/10.1042/BJ20041354

Kollau A, Beretta M, Russwurm M, Koesling D, et al. (2009). Mitochondrial nitrite reduction coupled to soluble guanylate cyclase activation: lack of evidence for a role in the bioactivation of nitroglycerin. Nitric Oxide 20: 53-60. http:// dx.doi.org/10.1016/j.niox.2008.09.003

Li J, Zhang M, Yang C, Dun Y, et al. (2009). Nitroglycerin protects small intestine from ischemia-reperfusion injury via NO-cGMP pathway and upregulation of alpha-CGRP. J. Gastrointest. Surg. 13: 478-485. http://dx.doi.org/10.1007/ s11605-008-0728-Z 
Luckey SW and Petersen DR (2001). Metabolism of 4-hydroxynonenal by rat Kupffer cells. Arch. Biochem. Biophys. 389: 77-83.http://dx.doi.org/10.1006/abbi.2001.2307

Madhusoodanan KS and Murad F (2007). NO-cGMP signaling and regenerative medicine involving stem cells. Neurochem. Res. 32: 681-694.http://dx.doi.org/10.1007/s11064-006-9167-y

Mayer B and Beretta M (2008). The enigma of nitroglycerin bioactivation and nitrate tolerance: news, views and troubles. Br. J. Pharmacol. 155: 170-184. http://dx.doi.org/10.1038/bjp.2008.263

McGuire JJ, Anderson DJ, McDonald BJ, Narayanasami R, et al. (1998). Inhibition of NADPH-cytochrome P450 reductase and glyceryl trinitrate biotransformation by diphenyleneiodonium sulfate. Biochem. Pharmacol. 56: 881-893. http:// dx.doi.org/10.1016/S0006-2952(98)00216-0

Meah Y, Brown BJ, Chakraborty S and Massey V (2001). Old yellow enzyme: reduction of nitrate esters, glycerin trinitrate, and propylene 1,2-dinitrate. Proc. Natl. Acad. Sci. USA 98: 8560-8565.http://dx.doi.org/10.1073/pnas.151249098

Munzel T (2008). [Recent findings on nitrates: their action, bioactivation and development of tolerance]. Dtsch. Med. Wochenschr. 133: 2277-2282.

Munzel T, Daiber A and Mülsch A (2005). Explaining the phenomenon of nitrate tolerance. Circ. Res. 97: 618-628. http:// dx.doi.org/10.1161/01.RES.0000184694.03262.6d

Ohsawa I, Nishimaki K, Yasuda C, Ohta S, et al. (2003). Deficiency in a mitochondrial aldehyde dehydrogenase increases vulnerability to oxidative stress in PC12 cells. J. Neurochem. 84: 1110-1117.

Ohta S and Ohsawa I (2006). Dysfunction of mitochondria and oxidative stress in the pathogenesis of Alzheimer's disease: on defects in the cytochrome c oxidase complex and aldehyde detoxification. J. Alzheimers Dis. 9: 155-166.

Tsou PS, Addanki V, Haas JA, Page NA, et al. (2009). Role of glutaredoxin-mediated protein S-glutathionylation in cellular nitroglycerin tolerance. J. Pharmacol. Exp. Ther. 329: 649-656. http://dx.doi.org/10.1124/jpet.108.149997

Wang MF, Han CL and Yin SJ (2009). Substrate specificity of human and yeast aldehyde dehydrogenases. Chem. Biol. Interact. 178: 36-39. http://dx.doi.org/10.1016/j.cbi.2008.10.002

Zhang J, Chen Z, Cobb FR and Stamler JS (2004). Role of mitochondrial aldehyde dehydrogenase in nitroglycerininduced vasodilation of coronary and systemic vessels: an intact canine model. Circulation 110: 750-755. http:// dx.doi.org/10.1161/01.CIR.0000138105.17864.6B 